\title{
A Synoptic Analysis of the Temporal and Spatial Aspects of Grapevine Leafroll Disease in a Historic Napa Vineyard and Experimental Vine Blocks
}

\author{
K. Arnold, D. A. Golino, and N. McRoberts
}

All authors: Department of Plant Pathology, and second author: Foundation Plant Services, University of California, Davis 95616. Accepted for publication 2 December 2016.

\begin{abstract}
Five Grapevine leafroll-associated virus 3 (GLRaV-3) epidemics were analyzed utilizing a standardized approach to robustly characterize the temporal and spatial parameters. Published data included in the analysis are from Spain, New Zealand, and Napa Valley, CA together with new data from a historic vineyard in Napa Valley, CA. Linear regression analyses of logittransformed incidence data indicated a maximum average increase of $11 \%$ per year in disease incidence, with considerable variation among locations. Spatial analyses, including distribution fitting, examination of the effective

sample size, and evaluation of the parameters of the binary power law fitted to variance data for disease incidence, indicated a high degree of consistency among the data sets. In all cases, except at very low disease incidence, a high degree of spatial aggregation was noted, with evidence that the degree of aggregation varied as a function of mean disease incidence. The polyetic dynamics of disease follow a logistic-like pattern over multiple seasons, consistent with limitation by inoculum availability (infected vines) at low incidence and limitation by disease-free vines at high incidence.
\end{abstract}

Grapevine leafroll disease (GLRD) is associated with a virus complex of grapevines and is graft transmissible. The economic impact of GRLD is estimated to be as high as \$226,405 per hectare over the life of a vineyard, depending upon the grape-growing region (Ricketts et al. 2015). GLRD is caused by at least five serologically distinct viruses, taxonomically referred to as Grapevine leafrollassociated virus- $1,-2,-3,-4$, and -7 (GLRaV-1,-2,-3,-4, and -7, respectively) in the Closteroviridae family. Virions in this family are typically long, flexuous rods, with the single-stranded RNA genome encapsidated in a modular coat protein. Because of the diversity of potential pathogen types, when referring to the viruses as a group, we will use GLRaV; when referring to the disease, GLRD will be used; and when referring to Grapevine leafroll-associated virus-3, GLRaV-3 will be used. GLRaV-1, -3 , and -4 are classified in the genus Ampelovirus, whereas GLRaV-2 is in the genus Closterovirus and GLRaV-7 is considered to belong to the tentative genus Velarivirus (Al Rwahnih et al. 2012). Within GLRaV-3, genetic variants have been demonstrated (Maree et al. 2015; Seah et al. 2012). Multiple species of GLRaV can be present in single vineyards and individual vines. Symptoms, although variable and dependent upon the grapevine variety, rootstock, and season, include delayed bud break and shoot development in spring, reduced yields, irregular ripening, and limited berry sugar accumulation. Additionally, infected red varieties may express the rolling under of leaf edges and reddening of the interveinal areas while the primary and secondary veins remain green; or, in white varieties, interveinal areas may become chlorotic from midsummer into fall (Alley et al. 1963; Goheen and Cook 1959; Goheen et al. 1959; Hale and Woodham 1979; Hewitt et al. 1962; Lee and Martin 2009; Lider et al. 1975; Maree et al. 2013; Martelli et al. 2012; Naidu et al. 2015). The California grapevine industry has grown increasingly concerned with the wide distribution of GLRaV-3 (Sharma et al. 2011), in part due to evidence that this virus is actively spreading (Golino et al. 2008).

Corresponding author: N. McRoberts; E-mail address: nmcroberts@ucdavis.edu

*The $\boldsymbol{e}$-Xtra logo stands for "electronic extra" and indicates that one supplementary note is published online.

(c) 2017 The American Phytopathological Society
GLRD was likely imported to the United States in infected propagation material from the "Old World" in the 1800s and was further distributed via commerce (Alley and Golino 2000; Maree et al. 2013). Early foresight in response to the demand for diseasedtested, professionally identified grape scion and rootstock varieties led to the establishment of the California Grapevine Registration and Certification (CGR\&C) program in the 1940s and 1950s (Alley and Golino 2000; Golino et al. 2008; Olmo 1951). The success of this program, in conjunction with U.S. regulated quarantines, has led to the removal of many viruses of grapevine in source material by methods such as heat therapy and microshoot tip tissue culture (Bostock et al. 2014; Gambino et al. 2006; Maree et al. 2013; Panattoni and Triolo 2010). Even though the estimated economic benefits to the industry are considerable, as much as $\$ 50$ million per year in the north coast region of California alone (Fuller et al. 2015), certification is voluntary and many growers still use noncertified planting material in their vineyards (Golino et al. 2008). This noncertified material can be a source of inoculum for the potential infection of newly established, certified vineyards.

GLRaV-3 is readily transmitted by multiple species of mealybugsgrape (Pseudococcus maritimus) obscure (P. vibruni), longtailed (P. longispinus), vine (Planococcus ficus), and citrus (P. citri Rossi)and some scale insects, all of which are present in California grapegrowing regions. The biology of the vector adds complexity to the epidemiology of GLRaV-3. Mealybugs transmit the virus in a semipersistent manner (Tsai et al. 2008), and earlier instars are typically more active in terms of crawling but do not have wings. Adult males, on the other hand, have wings but do not feed, suggesting that short-distance spread of this virus is dictated by crawling juveniles whereas long-distance spread is likely due to mealybugs carried by wind, equipment, and clothing (Almeida et al. 2013; Daane et al. 2012; Tsai et al. 2008, 2010).

Although many growers in California are prepared to collaboratively manage this disease via rogueing of newly planted certified blocks and mealybug suppression (Almeida et al. 2013), previous epidemiological studies from Australia, New Zealand, Spain, and Napa Valley, CA are inconclusive as to the factors that determine the temporal rate of increase in GLRaV-3 in vine blocks, with rates of increase varying greatly among and within grape-growing regions (Cabaleiro and Segura 1997a, 2006; Cabaleiro et al. 2008; Golino 
et al. 2008; Habili and Nutter 1997; Jordan 1993). This variability limits grower confidence in the cost-effectiveness of GLRaV-3 management.

As part of a wider program of support on vine health management, we present here the first comprehensive analyses of GLRaV-3 epidemiological characteristics. In addition to allowing disparate individual studies to be compared directly, our analyses provide new results on the general spatial characteristics of GLRaV-3 epidemics and estimates of spatial parameters that can be used in developing disease sampling protocols with known operating characteristics. Aside from regional breadth, these studies also span a time period from the early 1990s to the early 2010s. Our analysis places GLRaV-3 in a wider context of results from a range of studies of plant disease incidence (Hughes et al. 1997; Madden and Hughes 1995; McRoberts et al. 2003), allowing the characteristics of the disease to be assimilated into the wider body of knowledge on plant virus disease epidemiology.

\section{MATERIALS AND METHODS}

An exhaustive search of the literature for all previously published data was performed. All previously published data that included mapped disease incidence at the level of individual vines were reanalyzed in this study. Additionally, several data sets were provided by a historic vineyard in Napa Valley, CA, which have never previously been published.

Source of plant material (previously published vineyards). Beluso (Bueu, Pontevedra, Spain). Data for the Beluso vineyard were recovered from Figure 1 in Cabaleiro et al. (2008). These observations were based on 40 leafroll indicator plants (20 'Pinot Noir' and 20 'Cabernet Sauvignon') grafted onto 161.46C rootstocks planted between two previously GLRaV-3-infected grapevines. Disease incidence data were collected at eight time points over a span of 9 years. Vines were screened for GLRaV-3 using a double-antibody sandwich or direct immunoprinting enzyme-linked immunosorbent assay (DAS- or DIP-ELISA) every year between 1996 and 2007. Authors stated the presence of citrus mealybug (P. citri), which produces several overlapping generations in Galicia, Spain (Cabaleiro et al. 2008).

Meaño (Pontevedra, Spain). Data for the Meaño study were recovered from Figure 2 in Cabaleiro et al. (2008). Observations were based on 160 scions from four virus-free 'Albariño' clones, grafted onto 196.17C or SO4 virus-free certified rootstocks planted in 1988. Disease development was studied between 1992 and 1996 and again from 2003 to 2005 (Cabaleiro and Segura 1997a; Cabaleiro et al. 2008). All plants in the plot were screened on an annual basis for GLRaV-3 using DAS-ELISA. Authors stated no mealybugs were identified in the vineyard during the duration of this previous study (Cabaleiro et al. 2008).

Auckland. Data for the Auckland study was recovered from Figure 1 in Jordan (1993). This block was planted with Cabernet Sauvignon vines, and was originally described as having a randomly distributed initial infection arising from use of contaminated scion wood. Subsequent infections were thought, by the original authors, to be due to vector activity from within the block and outside the block. Visual symptoms of GLRaV-3 were monitored each year from 1988 to 1992.

Oakville (Napa, CA). The Oakville data were previously described by Golino et al. (2008). The vineyard was planted in 1989 using a certified rootstock field-budded with scion wood of Cabernet Sauvignon from an asymptomatic but not certified vineyard source. Symptoms associated with GLRD were not observed for the first 9 to 10 years, suggesting that original stock was free of virus. Monitoring of GLRaV-3 began in 2002 and ended in 2006. A qualitative description of disease progress within the block was reported by Golino et al. (2008). In total, 75 vine petiole samples were tested using ELISA for four viruses (GLRaV-1,-2,-3, and -4) and results demonstrated that only GLRaV-3 was present in symptomatic vines tested. Using their symptom scoring system, 35 samples were from vines rated strongly positive for leafroll, 20 vines rated negative for leafroll, and 20 vines rated questionable. The visual-symptom ratings were highly accurate, although not in perfect agreement with ELISA testing. All strong positive samples and questionable samples tested positive for GLRaV-3 with ELISA, while 2 of the 20 negative samples tested positive for GLRaV-3. These two visual false negatives were likely new infections from the year in which visual symptoms were recorded. At the time of data collection grape mealybug was documented in this vineyard (Golino et al. 2002, 2008).

Source of plant material (previously unpublished vineyards). Private vineyard blocks. Visual disease incidence data collected by a historic vineyard in the Napa Valley of California from 2007 to 2011 were provided for analysis. Scion wood used in these blocks originated from mother vines that were selected from highly monitored, asymptomatic and periodically tested mother vines and field budded onto certified rootstocks. Additionally, after planting, vineyard blocks were carefully monitored for GLRaV-3-associated symptoms, and new blocks did not show symptoms for many years after planting. Petiole samples collected in October 2012 were screened for GLRaV-3 in order to assess the accuracy of the visual observations. Using a simple random sampling procedure, 62 symptomatic vines that were marked as positive (by visual symptoms) on vineyard management maps were selected as positives for symptom verification, and 79 asymptomatic vines marked as negatives on the maps were randomly selected as negatives for verification. Four petioles were collected from each selected vine in order to control for potential uneven virus distribution within the vine.

Vines were assayed for GLRaV-3 using quantitative reversetranscription polymerase chain reaction (qRT-PCR) (TaqMan; Applied Biosystems Inc., Foster City, CA). Pooled petioles from plants $(200 \mathrm{mg})$ were homogenized in 1:10 guanadine buffer $(4 \mathrm{M}$ guanidine isothiocyanate, $0.2 \mathrm{M}$ sodium acetates [pH 5.0], $0.025 \mathrm{M}$ EDTA, and 2.5\% [wt/vol] PVP-40) using a Bioreba Homex 6 grinder (STA Labs, Legmont, CO). Total RNA was extracted from the lysate of homogenized material using the RNeasy Mini Plant kit and Qiashredder columns (Qiagen, Valencia, CA). Total purified RNA $(2 \mu \mathrm{l})$ was used in a $25-\mu \mathrm{l}$ final volume for one-step RT-PCR and $2 \mu \mathrm{l}$ of the same RNA was used in a 12- $\mu$ l final volume for qRT-PCR (Osman and Rowhani 2008; Osman et al. 2007). The qRT-PCR primers or TaqMan probes (MWG Inc., Biotech, NC) used in these analyses were the same as those used by Klaassen et al. (2011) for GLRaV-3. An 18S ribosomal RNA qRT-PCR assay (Osman et al. 2007) was used to verify that the total RNA samples could be amplified. qRT-PCR assays were run in duplicate or triplicate, and data were analyzed quantitatively by measuring the cycle threshold and graphically by an amplification plot.

In order to respect grower confidentiality, data from these private vineyard blocks are encoded as follows. PV1 and PV2 represent two adjacent private vineyards, both under the same management and ownership. B-number represents an individual block. For example, PV1B5 translates to private vineyard 1, block 5. In some cases, vineyard blocks that were subdivided by roads but managed as single units, or which occupy single blocks of ground but are identified by the vineyard owners by individual labels, were pooled to form larger blocks of data for analysis. Grape mealybug was present in this vineyard during data collection.

Temporal analysis. In order to describe the temporal aspects of the natural distribution of GLRaV-3 in Australia, New Zealand, Spain, and Napa Valley, CA, disease incidence (the proportion of infected vines in a block) was calculated for each site based on the observation sequence of the data. Only vineyard blocks consisting of four or more consecutive observations were included, resulting in 10 datasets. Data were transformed to logits on a natural log scale, and a linear regression of the logit (incidence) against time was performed to estimate the apparent infection rate of a logistic process. The $\mathrm{R}$ code (version 2.14.1, 32 bit) and data for this are available in the Supplementary Material. 
Spatial analysis. Multiple spatial analyses were performed in order to characterize the spatial pattern of GLRaV-3. For this purpose, each block was divided into sampling units or quadrats of $n=4$; this number was chosen due to the high variability in the size of the vineyard blocks included in the analyses. A quadrat is defined here as a two-by-two square consisting of two adjacent vines within each of two adjacent rows of vines. Nonrectangular blocks were modified by removing last rows or columns in order to adjust the field data for quadrat analysis.

Distribution analysis. The fits of observed frequencies of symptomatic vines per quadrat to the binomial and $\beta$-binomial distribution were examined using a distribution-fitting program in SAS, version 9.3, written by L. V. Madden (http://www.oardc.ohio-state. edu/pp702/Downloads.htm). The presence of spatial aggregation of disease is identified by a lack of fit of the observed frequencies of symptomatic plants per quadrat to a binomial distribution and a better fit to the expected frequencies for a $\beta$-binomial distribution. The degree of aggregation (or patchiness) is quantified by the aggregation parameter of the $\beta$-binomial distribution, typically denoted $\theta$. In cases where attribution of a dataset as either binomial or $\beta$-binomial was difficult, typically a problem at low mean disease incidence, the corrected Akaike Information Criterion (AICc) was used for choosing one distribution over the other on the basis of the smaller of the two AICc values (Burnham et al. 2011; Hughes and Madden 1992, 1993; Hughes et al. 1996, 1997; Madden and Hughes 1994, 1995; Turechek and McRoberts 2013).

Binary power law analyses. Parameters for the binary power law were obtained by regression using 67 data points, after discarding one dataset with a disease incidence of zero. The binary power law can be written as (Madden et al. 2007)

$$
s_{y}^{2}=A\left(s_{\text {bin }}^{2}\right)^{b}
$$

in which $s_{y}^{2}$ is the observed quadrat variance, $s_{\text {bin }}^{2}$ is the binomial quadrat variance, and $A$ and $b$ are estimated coefficients from the linear regression analysis:

$$
\log _{10}\left(s_{y}^{2}\right)=\log _{10} a+b \log _{10}(\bar{y}[1-\bar{y}] / n)
$$

in which $A=a n^{b}$; therefore

$$
\log _{10}\left(s_{y}^{2}\right)=\log _{10} A+b \log _{10}\left(s_{\text {bin }}^{2}\right)
$$

$\log (A)$ and $b$ were estimated from the linear regression of the observed variance of vine disease incidence on the theoretical (binomial) variance. The $\mathrm{R}$ code and data used for the analysis are available online in Supplementary Note S1. In equation 3, when $b$ and $A$ both equal 1, a binomial distribution is described, whereas if $b=1$ and $A>1$, aggregation is present in the system, although the $\beta$-binomial parameter $\theta$ is constant. If both $b$ and $A$ are greater than 1 ,

TABLE 1. Linear regression of the logit disease incidence data are shown here $^{\mathrm{a}}$

\begin{tabular}{lcccccrc}
\hline & $y$-int & $P$ & $r$ & $P$ & $R^{2}$ & $F$ & $P$ \\
\hline Oakville & -1.42 & 0.008 & 0.41 & 0.008 & 0.92 & \multicolumn{1}{c}{35.8} & 0.009 \\
Beluso & -2.84 & 0.000 & 0.66 & 0.000 & 0.97 & 162.90 & 0.000 \\
Meano & -1.59 & 0.001 & 0.23 & 0.004 & 0.99 & 265.90 & 0.004 \\
Auckland & -3.56 & 0.002 & 1.10 & 0.002 & 0.96 & 91.05 & 0.002 \\
PV1B5 & -2.32 & 0.000 & 0.07 & 0.020 & 0.87 & 20.74 & 0.020 \\
PV1B212223 & -1.04 & 0.024 & 0.25 & 0.052 & 0.90 & 17.73 & 0.052 \\
PV1B24 & -4.71 & 0.001 & 0.40 & 0.027 & 0.95 & 35.91 & 0.027 \\
PV1B7AB & -4.39 & 0.002 & 0.33 & 0.086 & 0.68 & 6.36 & 0.086 \\
PV1B7C & -4.92 & 0.002 & 0.40 & 0.059 & 0.75 & 8.86 & 0.059 \\
PV1B6A1 & -2.87 & 0.002 & 0.19 & 0.046 & 0.91 & 20.45 & 0.046 \\
\hline
\end{tabular}

a Auckland expressed the greatest slope of 1.10, whereas the minimum slope of 0.07 is associated with PV1B5. The average of all slopes is 0.40 . $P$ represents the corresponding significance $P$ values. aggregation is present in the system, and the $\beta$-binomial parameter $\theta$ changes as a function of mean disease incidence (Hughes and Gottwald 1999).

Spatial hierarchy. The spatial structure of GLRaV-3 epidemics was explored further by looking at the relationship between disease incidence at the scale of individual vines and at the scale of quadrats (Hughes et al. 1997; McRoberts et al. 2003).

If the pattern of diseased vines is random and vines are sampled using quadrats of $n$ vines, the theoretical relationship between disease incidence at the quadrat scale and the scale of an individual vine is given by:

$$
I_{\text {high }}=1-\left(1-I_{\text {low }}\right)^{n}
$$

in which $I_{\text {low }}$ is disease incidence and $I_{\text {high }}$ is quadrat incidence (McRoberts et al. 2003). The theoretical range of $\mathrm{I}_{\text {low }}$ is from 0 to 1 , allowing the theoretical $I_{\text {high }} I_{\text {low }}$ relationship to be calculated for any value of $n$. Spatial aggregation in observed data collected using quadrats of size $n$ is evident when the observed data points lie below the curve for the theoretical relationship when $I_{\text {high }}$ is plotted on the ordinate and $I_{\text {low }}$ is plotted on the abscissa.

Effective sample size estimation. In the case where observed data lie systematically below the curve depicting the $I_{\text {high }}-I_{l o w}$ relationship, as if on an equivalent curve $I_{\text {high }}=1-\left(1-I_{\text {low }}\right)^{\gamma}$, with $\gamma<n$, an estimate of the value of $\gamma$ can be obtained by linear regression from the equation (McRoberts et al. 2003)

$$
\operatorname{CLL}\left(I_{\text {high }}\right)=\ln (\gamma)+\operatorname{CLL}\left(I_{\text {low }}\right)
$$

in which $\operatorname{CLL}(\bullet)$ is the complementary log-log transform and $y=$ $\ln [-\ln (1-x)]$. The value of $\gamma$ was estimated by regression from 61 data points after seven datasets were discarded because $I_{\text {high }}$ or $I_{\text {low }}$ values were either 0 or 1 . The Data and $\mathrm{R}$ program used for estimating the CLL relationship are available in the Supplementary Material.

\section{RESULTS}

Temporal analyses. All blocks consisted of either highly monitored vines or certified material as mother material, and GLRaV-3 epidemics in the available data progressed over several years in each block from initially low levels (in some cases 0 ), with the exception of the Oakville dataset in which the disease incidence at the vine scale was already 0.27 when observations started.

The sequence of observations was longest at Beluso, where disease progress was followed for eight seasons. Many of the other data sets consisted of series of four or five annual observations. The results of linear regression of the logit disease incidence data to obtain estimates of the logistic rate parameter $(r)$ are shown in Table 1.

Given the extremely short series of data in most cases, these results should be viewed as a way to obtain a comparative value of the rate of each epidemic that is meaningful in a wider epidemiological context, rather than a formal statistical attempt to fit a model to a data set. Bearing in mind the limitations of the analyses, the highest value of $r$ was found in the Auckland data at $r=1.10$ logits/year $\left(R^{2}=0.96, F=91.05\right)$, the minimum value in the historic vineyard in Napa at $r=0.07$ logits/year $\left(R^{2}=0.87, F=20.74\right)$, and the mean across all datasets was $r=0.43$ logits/year.

Writing $p$ for the proportion of vines diseased per year, and assuming that all epidemics would result in $100 \%$ disease incidence eventually, the rate equation for the logistic growth model is given by the familiar rate equation:

$$
(d p / d t)=r p(1-p)
$$

Assuming logistic dynamics of GLRaV-3 documented by Habili and Nutter (1997), the average maximum rate for any given epidemic is $r=0.43 \times 0.50(1-0.50)$, which is approximately 0.11 or an $11 \%$ increase in infected vines per year (Madden et al. 2007). 
Disease progress of GLRD through observation periods in multiple regions is shown in Figure 1.

Spatial analysis. Distribution analyses. In total, 69 locationyears of data were available for distributional analysis. Maximumlikelihood estimates for the parameters of the binomial and $\beta$-binomial were obtained indicating that the $\beta$-binomial was a better fit in 52 cases ( $75 \%$ of datasets) and the binomial in 17 cases $(25 \%$ of datasets). In all cases when the maximum-likelihood estimate of $\theta$ could not be successfully calculated, the associated $\mathrm{AIC}_{\mathrm{C}}$ (Burnham et al. 2011) value indicated that the binomial distribution was the better fit to the data than the $\beta$-binomial distribution (Table 2).

Values of the aggregation parameter $\theta$ are plotted against mean disease incidence in Figure 2. The theoretical line for $\theta$ as a function of mean disease incidence, based on the estimated parameters of the binary power law (see below), is also shown in Figure 2.

Binary power law analyses. The linear regression for the binary power law analyses resulted in a linear relationship expressed by the formula:

$$
\log _{10}\left(s_{y}^{2}\right)=0.477+(1.162) \log _{10}\left(s_{\text {bin }}^{2}\right)
$$

with $R^{2}=0.96, P<0.0001$. In terms of the binary power law analysis:

$$
\log _{10}\left(s_{y}^{2}\right)=\log _{10} A+b \log _{10}\left(s_{\text {bin }}^{2}\right)
$$

in which $A=a n^{b}, A=10^{0.477}=3.00$, and $b=1.162$. These values indicate the presence of aggregation, which varies as a function of the mean (Hughes and Madden 1992, 1993). The data and fitted regression line are shown in Figure 3. The high variance accounted for using a single binary power law regression is consistent with the action of similar spatial processes across the wide range of conditions in the different datasets.

Spatial hierarchy. The results of the spatial hierarchy analysis can be seen in Figure 4. The observed data lie below the theoretical line for the binomial relationship for group size $n=4$, indicating the presence of aggregation, consistent with the results of the distributionfitting and binary power law analyses. Fitting the CLL regression model between quadrat and vine disease incidence data resulted in the relationship $y=0.741+0.877 x\left(R^{2}=0.969, P<0.0001\right)$, from which the value of the effective sample size, $\gamma$, is $e^{0.74}=2.09$. The effective sample size represents the number of apparent statistically independent observations in each sample under the assumptions that the binomial distribution is an appropriate statistical model for the data. When it is less than the actual sample size, $n$, it provides an index of the degree to which the disease status of individual vines in a sampling quadrat is not independent (Hughes and Gottwald 1999; Hughes et al. 1997; McRoberts et al. 2003). The estimated relationship is shown in Figure 5: $\operatorname{CLL}\left(I_{\text {low }}\right)=\ln (2.09)+\operatorname{CLL}\left(I_{\text {high }}\right)$ in CLL form.

\section{DISCUSSION}

One of the most striking outcomes of the analyses conducted in this study is the contrast between the diversity observed in the temporal rate of disease increase and the level of conformity of the spatial analyses to a single, common, binary power law model. Longer sets of data available suggested that the polyetic dynamics of GLRaV-3 are logistic, supporting an earlier analysis of a single data set by Habili and Nutter (1997). In fact, if inoculum (or, more generally, force of infection) is proportional to the incidence of infection, regardless of the dispersal process, disease increase in a fixed population of hosts will show logistic-like dynamics (Hughes and McRoberts 2014). On this basis, we calculated estimates of logistic rate parameters for epidemics with four or more observations over time. The estimated values ranged from a maximum of 1.10 logit/year for the data from Auckland, NZ reported by Jordan (1993) to a minimum of 0.07 logit/year in one of the previously unpublished datasets collected in a historic vineyard in Napa Valley, CA.

The observed variability in the apparent infection rate among the studies from different locations, without the benefit of accompanying analyses of the spatial properties of these epidemics, would be difficult to interpret in terms of a common dispersal mechanism. However, although the individual GLRaV-3 epidemics progressed at a wide range of different speeds, the spatial analysis clearly shows that all the epidemics lie on the same incidence-incidence curve and conform to a common regression relationship of the (log) observed variance on the (log) binomial variance. This is strong evidence that the dispersal processes operating at the different locations have the same biophysical properties because the incidence-incidence curve and binary power law are expressions of dispersal processes (Hughes

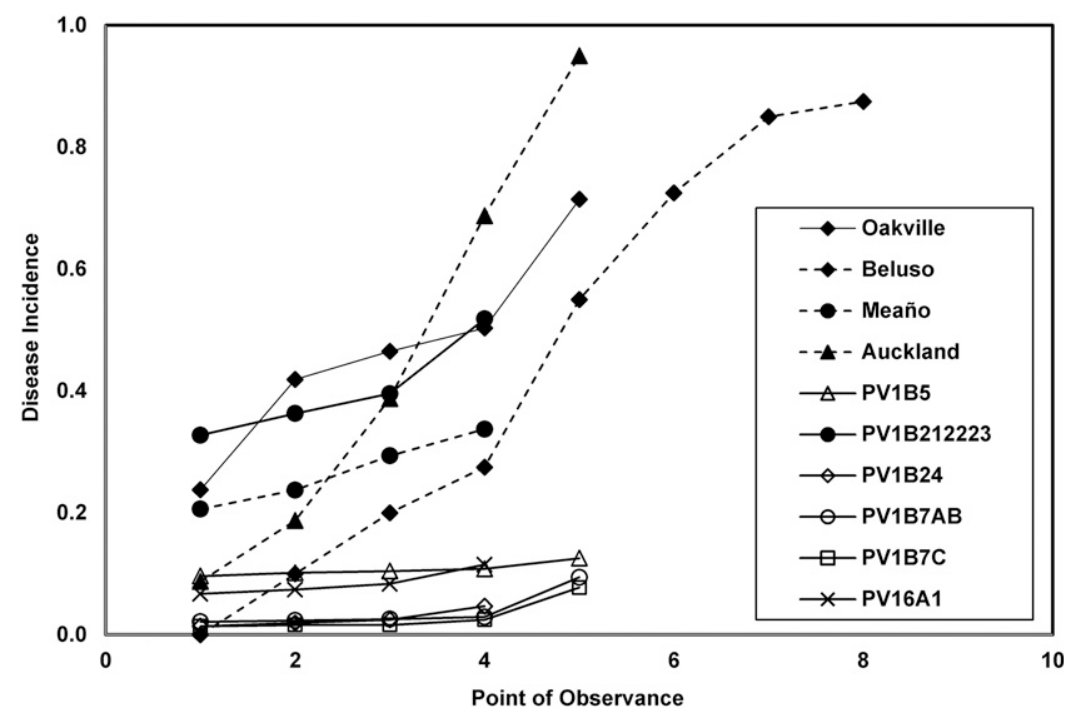

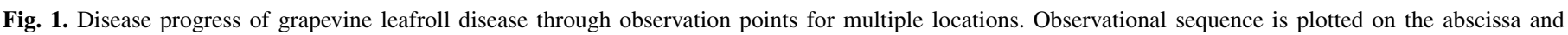

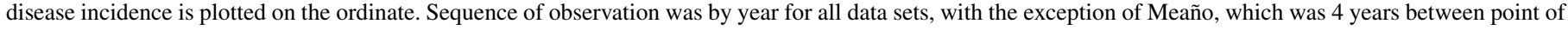

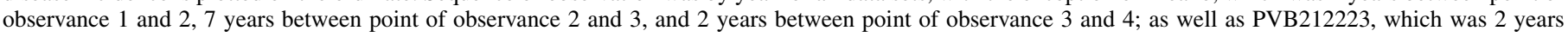

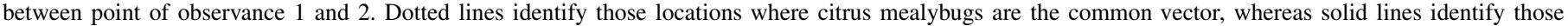
locations where grape mealybugs are the common vector. 
TABLE 2. Each data set was fitted to both the binomial and $\beta$-binomial for best fit ${ }^{\mathrm{a}}$

\begin{tabular}{|c|c|c|c|c|c|c|c|c|c|c|c|c|c|c|}
\hline \multicolumn{4}{|c|}{ Block details } & \multicolumn{4}{|c|}{ Binomial } & \multicolumn{7}{|c|}{$\beta$-Binomial } \\
\hline Location & Planted & Observed & $p$ (a priori) & $\mathrm{AICc}$ & $P$ & $p \mathrm{SE}$ & $\operatorname{Pr}>|t|$ & $\mathrm{AICc}$ & $\theta$ & $\theta \mathrm{SE}$ & $\operatorname{Pr}>|t|$ & $P$ & $p \mathrm{SE}$ & $\operatorname{Pr}>|t|$ \\
\hline Auckland & 1985 & 1988 & 0.088 & 35.80 & 0.088 & 0.032 & 0.012 & 35.50 & 0.234 & 0.262 & 0.000 & 0.088 & 0.040 & 0.038 \\
\hline Auckland & 1985 & 1989 & 0.188 & 52.90 & 0.188 & 0.044 & 0.000 & 51.50 & 0.269 & 0.222 & 0.240 & 0.186 & 0.056 & 0.003 \\
\hline Auckland & 1985 & 1990 & 0.388 & 68.10 & 0.388 & 0.054 & $<0.0001$ & 66.40 & 0.270 & 0.197 & 0.185 & 0.388 & 0.070 & $<0.0001$ \\
\hline Auckland & 1985 & 1991 & 0.688 & 58.70 & 0.688 & 0.052 & $<0.0001$ & 60.30 & 0.105 & 0.135 & 0.445 & 0.688 & 0.059 & $<0.0001$ \\
\hline Auckland & 1985 & 1992 & 0.950 & 22.90 & 0.950 & 0.024 & $<0.0001$ & 25.60 & $*$ & $*$ & $*$ & 0.937 & 0.000 & $<0.0001$ \\
\hline Beluso & 1996 & 1998 & 0.100 & 23.00 & 0.100 & 0.047 & 0.061 & 20.80 & 0.769 & 0.891 & 0.409 & 0.096 & 0.069 & 0.197 \\
\hline Beluso & 1996 & 1999 & 0.200 & 33.40 & 0.200 & 0.063 & 0.010 & 29.30 & 0.887 & 0.826 & 0.308 & 0.211 & 0.101 & 0.063 \\
\hline Beluso & 1996 & 2000 & 0.275 & 37.70 & 0.275 & 0.071 & 0.003 & 33.00 & 0.820 & 0.661 & 0.243 & 0.277 & 0.108 & 0.028 \\
\hline Beluso & 1996 & 2001 & 0.550 & 32.10 & 0.550 & 0.079 & $<0.0001$ & 35.10 & 0.065 & 0.167 & 0.705 & 0.551 & 0.086 & $<0.0001$ \\
\hline Beluso & 1996 & 2002 & 0.725 & 30.50 & 0.725 & 0.071 & $<0.0001$ & 32.80 & 0.166 & 0.234 & 0.494 & 0.727 & 0.084 & $<0.0001$ \\
\hline Beluso & 1996 & 2003-2004 & 0.850 & 23.60 & 0.850 & 0.056 & $<0.0001$ & 26.30 & 0.128 & 0.239 & 0.605 & 0.851 & 0.065 & $<0.0001$ \\
\hline Beluso & 1996 & $2005-2006$ & 0.875 & 20.70 & 0.875 & 0.052 & $<0.0001$ & 23.90 & 0.012 & 0.151 & 0.940 & 0.875 & 0.053 & $<0.0001$ \\
\hline Meano & 1989 & 1992 & 0.206 & 98.00 & 0.206 & 0.032 & $<0.0001$ & 99.20 & 0.072 & 0.086 & 0.407 & 0.206 & 0.035 & $<0.0001$ \\
\hline Meano & 1989 & 1996 & 0.238 & 107.70 & 0.238 & 0.034 & $<0.0001$ & 107.20 & 0.124 & 0.100 & 0.219 & 0.238 & 0.039 & $<0.0001$ \\
\hline Meano & 1989 & 2003 & 0.294 & 130.80 & 0.294 & 0.036 & $<0.0001$ & 119.40 & 0.377 & 0.176 & 0.038 & 0.297 & 0.049 & $<0.0001$ \\
\hline Meano & 1989 & 2005 & 0.338 & 133.30 & 0.338 & 0.037 & $<0.0001$ & 124.20 & 0.321 & 0.152 & 0.041 & 0.340 & 0.049 & $<0.0001$ \\
\hline Oakville & 1989 & 2002 & 0.238 & $14,501.00$ & 0.238 & 0.003 & $<0.0001$ & $8,926.80$ & 1.908 & 0.084 & $<0.0001$ & 0.240 & 0.006 & $<0.0001$ \\
\hline Oakville & 1989 & 2003 & 0.419 & $17,325.00$ & 0.430 & 0.004 & $<0.0001$ & $11,479.00$ & 1.603 & 0.058 & $<0.0001$ & 0.436 & 0.007 & $<0.0001$ \\
\hline Oakville & 1989 & 2004 & 0.465 & $17,534.00$ & 0.514 & 0.004 & $<0.0001$ & $11,610.00$ & 1.613 & 0.058 & $<0.0001$ & 0.520 & 0.007 & $<0.0001$ \\
\hline Oakville & 1989 & 2005 & 0.503 & $17,289.00$ & 0.564 & 0.004 & $<0.0001$ & $11,513.00$ & 1.573 & 0.057 & $<0.0001$ & 0.569 & 0.007 & $<0.0001$ \\
\hline Oakville & 1989 & 2006 & 0.714 & $15,126.00$ & 0.714 & 0.004 & $<0.0001$ & $10,146.00$ & 1.451 & 0.058 & $<0.0001$ & 0.718 & 0.006 & $<0.0001$ \\
\hline PV16A1 & 1990 & 2007 & 0.067 & $1,253.30$ & 0.067 & 0.004 & $<0.0001$ & $1,226.50$ & 0.097 & 0.026 & 0.000 & 0.067 & 0.005 & $<0.0001$ \\
\hline PV16A1 & 1990 & 2009 & 0.074 & $1,330.80$ & 0.074 & 0.004 & $<0.0001$ & $1,303.20$ & 0.098 & 0.025 & 0.000 & 0.074 & 0.005 & $<0.0001$ \\
\hline PV16A1 & 1990 & 2010 & 0.084 & $1,440.80$ & 0.084 & 0.005 & $<0.0001$ & $1,400.80$ & 0.122 & 0.027 & $<0.0001$ & 0.083 & 0.005 & $<0.0001$ \\
\hline PV16A1 & 1990 & 2011 & 0.115 & $1,737.70$ & 0.115 & 0.005 & $<0.0001$ & $1,689.60$ & 0.128 & 0.025 & $<0.0001$ & 0.115 & 0.006 & $<0.0001$ \\
\hline PV1B10 & 2006 & 2011 & 0.004 & 74.20 & 0.004 & 0.001 & 0.008 & 76.20 & $*$ & $*$ & * & 0.004 & 0.000 & $<0.0001$ \\
\hline PV1B12 & 2006 & 2011 & 0.005 & 102.30 & 0.005 & 0.002 & 0.002 & 100.80 & 0.072 & 0.084 & 0.392 & 0.005 & 0.002 & 0.004 \\
\hline PV1B14 & 2001 & 2010 & 0.034 & 263.10 & 0.034 & 0.005 & $<0.0001$ & 265.10 & $*$ & $*$ & $*$ & 0.034 & $*$ & * \\
\hline PV1B14 & 2001 & 2011 & 0.224 & 848.50 & 0.224 & 0.012 & $<0.0001$ & 813.40 & 0.188 & 0.044 & $<0.0001$ & 0.224 & 0.014 & $<0.0001$ \\
\hline PV1B15 & 1998 & 2010 & 0.009 & 83.00 & 0.009 & 0.003 & 0.003 & 76.80 & 0.199 & 0.180 & 0.269 & 0.009 & 0.004 & 0.015 \\
\hline PV1B15 & 1998 & 2011 & 0.010 & 89.40 & 0.010 & 0.003 & 0.002 & 84.10 & 0.170 & 0.152 & 0.265 & 0.010 & 0.004 & 0.009 \\
\hline PV1B212223 & 1988 & 2007 & 0.328 & $7,128.10$ & 0.328 & 0.005 & $<0.0001$ & $6,378.90$ & 0.388 & 0.024 & $<0.0001$ & 0.328 & 0.007 & $<0.0001$ \\
\hline PV1B212223 & 1988 & 2009 & 0.363 & $7,600.30$ & 0.363 & 0.005 & $<0.0001$ & $6,584.90$ & 0.488 & 0.028 & $<0.0001$ & 0.364 & 0.007 & $<0.0001$ \\
\hline PV1B212223 & 1988 & 2010 & 0.396 & $7,653.50$ & 0.396 & 0.005 & $<0.0001$ & $6,722.90$ & 0.453 & 0.026 & $<0.0001$ & 0.397 & 0.007 & $<0.0001$ \\
\hline PV1B212223 & 1988 & 2011 & 0.518 & $7,661.90$ & 0.518 & 0.005 & $<0.0001$ & $6,883.30$ & 0.390 & 0.023 & $<0.0001$ & 0.519 & 0.007 & $<0.0001$ \\
\hline PV1B24 & 1999 & 2008 & 0.014 & 836.70 & 0.014 & 0.001 & $<0.0001$ & 831.70 & 0.033 & 0.102 & $<0.0001$ & 0.033 & 0.019 & 0.076 \\
\hline PV1B24 & 1999 & 2009 & 0.019 & $1,067.20$ & 0.019 & 0.002 & $<0.0001$ & $1,054.00$ & 0.049 & 0.020 & $<0.0001$ & 0.019 & 0.002 & $<0.0001$ \\
\hline PV1B24 & 1999 & 2010 & 0.025 & $1,273.10$ & 0.025 & 0.002 & $<0.0001$ & $1,248.50$ & 0.068 & 0.021 & 0.001 & 0.025 & 0.002 & $<0.0001$ \\
\hline PV1B24 & 1999 & 2011 & 0.047 & $1,960.30$ & 0.047 & 0.002 & $<0.0001$ & $1,953.50$ & 0.031 & 0.013 & 0.016 & 0.047 & 0.003 & $<0.0001$ \\
\hline PV1B25 & 2005 & 2011 & 0.025 & 796.70 & 0.025 & 0.002 & $<0.0001$ & 722.40 & 0.223 & 0.058 & 0.000 & 0.025 & 0.003 & $<0.0001$ \\
\hline PV1B252627 & 2005 & 2009 & 0.002 & 228.30 & 0.002 & 0.000 & $<0.0001$ & 219.40 & 0.081 & 0.064 & 0.210 & 0.002 & 0.000 & $<0.0001$ \\
\hline PV1B252627 & 2005 & 2010 & 0.010 & 855.90 & 0.010 & 0.001 & $<0.0001$ & 715.80 & 0.326 & 0.083 & $<0.0001$ & 0.009 & 0.001 & $<0.0001$ \\
\hline PV1B5 & 1994 & 2007 & 0.096 & $2,118.00$ & 0.096 & 0.005 & $<0.0001$ & $1,542.00$ & 0.899 & 0.109 & $<0.0001$ & 0.097 & 0.007 & $<0.0001$ \\
\hline PV1B5 & 1994 & 2008 & 0.102 & $2,158.10$ & 0.102 & 0.005 & $<0.0001$ & $1,630.30$ & 0.785 & 0.093 & $<0.0001$ & 0.103 & 0.007 & $<0.0001$ \\
\hline PV1B5 & 1994 & 2009 & 0.105 & $2,193.70$ & 0.105 & 0.005 & $<0.0001$ & $1,661.50$ & 0.782 & 0.092 & $<0.0001$ & 0.106 & 0.007 & $<0.0001$ \\
\hline PV1B5 & 1994 & 2010 & 0.108 & $2,229.00$ & 0.108 & 0.005 & $<0.0001$ & $1,706.90$ & 0.750 & 0.087 & $<0.0001$ & 0.109 & 0.007 & $<0.0001$ \\
\hline PV1B5 & 1994 & 2011 & 0.126 & $2,380.30$ & 0.126 & 0.005 & $<0.0001$ & $1,915.60$ & 0.615 & 0.068 & $<0.0001$ & 0.127 & 0.008 & $<0.0001$ \\
\hline PV1B7AB & 2002 & 2007 & 0.021 & $1,162.60$ & 0.022 & 0.002 & $<0.0001$ & $1,163.50$ & 0.011 & 0.012 & 0.357 & 0.022 & 0.002 & $<0.0001$ \\
\hline PV1B7AB & 2002 & 2008 & 0.024 & $1,237.90$ & 0.024 & 0.002 & $<0.0001$ & $1,231.30$ & 0.033 & 0.016 & 0.033 & 0.024 & 0.002 & $<0.0001$ \\
\hline PV1B7AB & 2002 & 2009 & 0.026 & $1,319.80$ & 0.026 & 0.002 & $<0.0001$ & $1,312.90$ & 0.034 & 0.015 & 0.028 & 0.026 & 0.002 & $<0.0001$ \\
\hline PV1B7AB & 2002 & 2010 & 0.029 & $1,439.40$ & 0.029 & 0.002 & $<0.0001$ & $1,435.80$ & 0.025 & 0.014 & 0.060 & 0.029 & 0.002 & $<0.0001$ \\
\hline PV1B7AB & 2002 & 2011 & 0.094 & $2,998.30$ & 0.094 & 0.003 & $<0.0001$ & $2,999.60$ & 0.008 & 0.010 & 0.432 & 0.094 & 0.003 & $<0.0001$ \\
\hline PV1B7C & 2002 & 2007 & 0.014 & 449.40 & 0.014 & 0.002 & $<0.0001$ & 449.30 & 0.023 & 0.022 & 0.300 & 0.014 & 0.002 & $<0.0001$ \\
\hline PV1B7C & 2002 & 2008 & 0.016 & 499.60 & 0.016 & 0.002 & $<0.0001$ & 500.50 & 0.015 & 0.019 & 0.417 & 0.016 & 0.002 & $<0.0001$ \\
\hline PV1B7C & 2002 & 2009 & 0.016 & 499.60 & 0.016 & 0.002 & $<0.0001$ & 500.50 & 0.015 & 0.019 & 0.417 & 0.016 & 0.002 & $<0.0001$ \\
\hline PV1B7C & 2002 & 2010 & 0.025 & 684.60 & 0.025 & 0.002 & $<0.0001$ & 682.40 & 0.029 & 0.019 & 0.136 & 0.025 & 0.003 & $<0.0001$ \\
\hline PV1B7C & 2002 & 2011 & 0.078 & $1,427.40$ & 0.078 & 0.004 & $<0.0001$ & $1,428.20$ & 0.015 & 0.014 & 0.309 & 0.078 & 0.004 & $<0.0001$ \\
\hline PV1B8 & 1989 & 2007 & 0.454 & $4,264.00$ & 0.454 & 0.007 & $<0.0001$ & $3,860.70$ & 0.366 & 0.029 & $<0.0001$ & 0.453 & 0.010 & $<0.0001$ \\
\hline PV2B1 & $* *$ & 2009 & 0.002 & 74.90 & 0.002 & 0.001 & 0.014 & 76.90 & $*$ & $*$ & * & 0.002 & 0.001 & 0.014 \\
\hline PV2B1 & $* *$ & 2010 & 0.017 & 485.40 & 0.017 & 0.002 & $<0.0001$ & 482.40 & 0.040 & 0.027 & 0.135 & 0.017 & 0.002 & $<0.0001$ \\
\hline PV2B2 & $* *$ & 2009 & 0.022 & 464.70 & 0.022 & 0.003 & $<0.0001$ & 373.20 & 0.470 & 0.149 & 0.002 & 0.022 & 0.004 & $<0.0001$ \\
\hline PV2B2 & $* *$ & 2010 & 0.031 & 601.50 & 0.031 & 0.003 & $<0.0001$ & 486.00 & 0.480 & 0.128 & 0.000 & 0.031 & 0.005 & $<0.0001$ \\
\hline PV2B3 & $* *$ & 2009 & 0.061 & 375.20 & 0.061 & 0.008 & $<0.0001$ & 246.70 & 1.204 & 0.372 & 0.001 & 0.060 & 0.012 & $<0.0001$ \\
\hline PV2B3 & $* *$ & 2010 & 0.130 & 424.80 & 0.073 & 0.008 & $<0.0001$ & 274.50 & 1.297 & 0.369 & 0.001 & 0.071 & 0.013 & $<0.0001$ \\
\hline PV2B4 & $* *$ & 2010 & 0.017 & 215.10 & 0.017 & 0.003 & $<0.0001$ & 212.50 & 0.065 & 0.051 & 0.205 & 0.017 & 0.003 & $<0.0001$ \\
\hline PV2B5 & $* *$ & 2009 & 0.037 & 562.50 & 0.037 & 0.004 & $<0.0001$ & 554.20 & 0.068 & 0.032 & 0.033 & 0.037 & 0.004 & $<0.0001$ \\
\hline PV2B5 & $* *$ & 2010 & 0.038 & 356.70 & 0.020 & 0.003 & $<0.0001$ & 355.30 & 0.040 & 0.031 & 0.205 & 0.020 & 0.003 & $<0.0001$ \\
\hline PV3B11 & 2011 & 2013 & 0.005 & 110.60 & 0.005 & 0.001 & 0.001 & 112.60 & $*$ & $*$ & $*$ & 0.005 & $*$ & $*$ \\
\hline PV3B12 & 2012 & 2013 & 0.017 & 284.60 & 0.017 & 0.003 & $<0.0001$ & 285.80 & 0.019 & 0.027 & 0.468 & 0.017 & 0.003 & $<0.0001$ \\
\hline
\end{tabular}

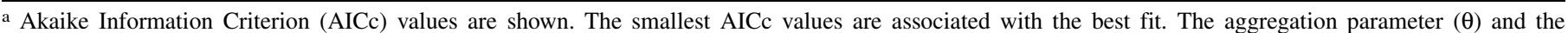
incidence $(p)$ are both shown for each data set, with the associated standard error (SE) and significance value of $P>t$. Values unable to be calculated are expressed with an asterisk $(*)$. Values not provided are represented by double asterisks $(* *)$. 
et al. 1997; McRoberts et al. 2003). When taken together, the temporal and spatial analyses indicate that what varies among the epidemics is the speed at which each epidemic progresses along the incidenceincidence curve from the $(0,0)$ point in the lower left of Figure 4 toward the $(1,1)$ point in the top right.

The analysis of spatial hierarchy places the different epidemics within a single, common set of spatiotemporal dynamics. This stable statistical property of GLRaV-3 epidemics is a function of

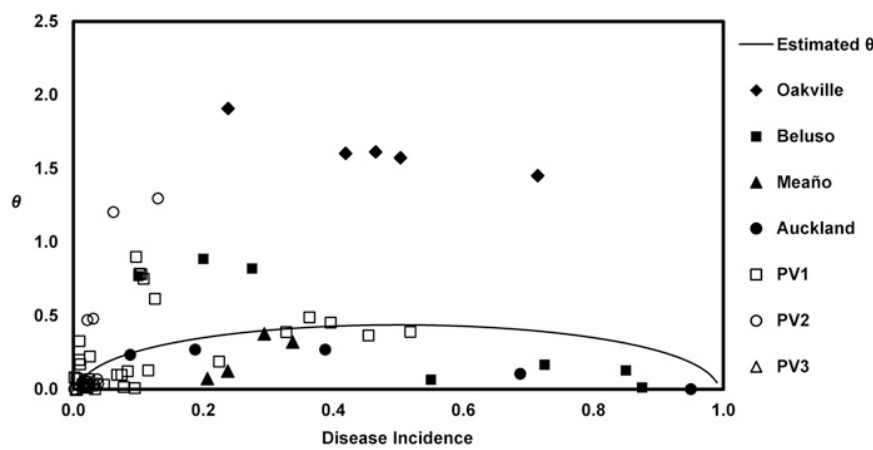

Fig. 2. Disease incidence is plotted on the abscissa and the aggregation parameter $(\theta)$ is plotted on the ordinate. A theoretical line representing the epidemic is plotted based on the estimates produced from the binary power law analysis. disease transmission characteristics as well as the basic biology of the vectors. Additionally, fitting the CLL regression model between quadrat and vine disease incidence data yielded an estimate of the effective sample size of $\gamma=2.09$, based on quadrats of $n=4$ vines, implying that the disease status of vines is highly correlated with that of their nearest neighbors in all of the data sets examined, covering a wide range of mean disease incidence.

Although the data in this study does not differentiate between variations in vector species fecundity, demographics, or virus transmission efficiency, further research on this topic might reveal why some vineyards experience a higher annual rate of disease increase than others. For example, it is known that some mealybug species are capable of producing as many as 10 overlapping generations per season under typical grape-growing conditions, whereas others produce only 2 to 3 discrete generations per season (Almeida et al. 2013; Cabaleiro and Segura 1997a,b; Daane et al. 2012; Golino et al. 2002; Sharma et al. 2011; Tsai et al. 2008). If disease transmission mainly occurs as a result of juveniles feeding and crawling between vines, these differences in the frequency of production of juvenile life stages would be likely to have consequences for the rate of disease increase, without altering the spatial properties of GLRaV-3 epidemics.

GLRaV-3 primary inoculum sources can result from infected planting material and viruliferous mealybugs from neighboring vineyard blocks. In polyetic epidemics such as these, each season,
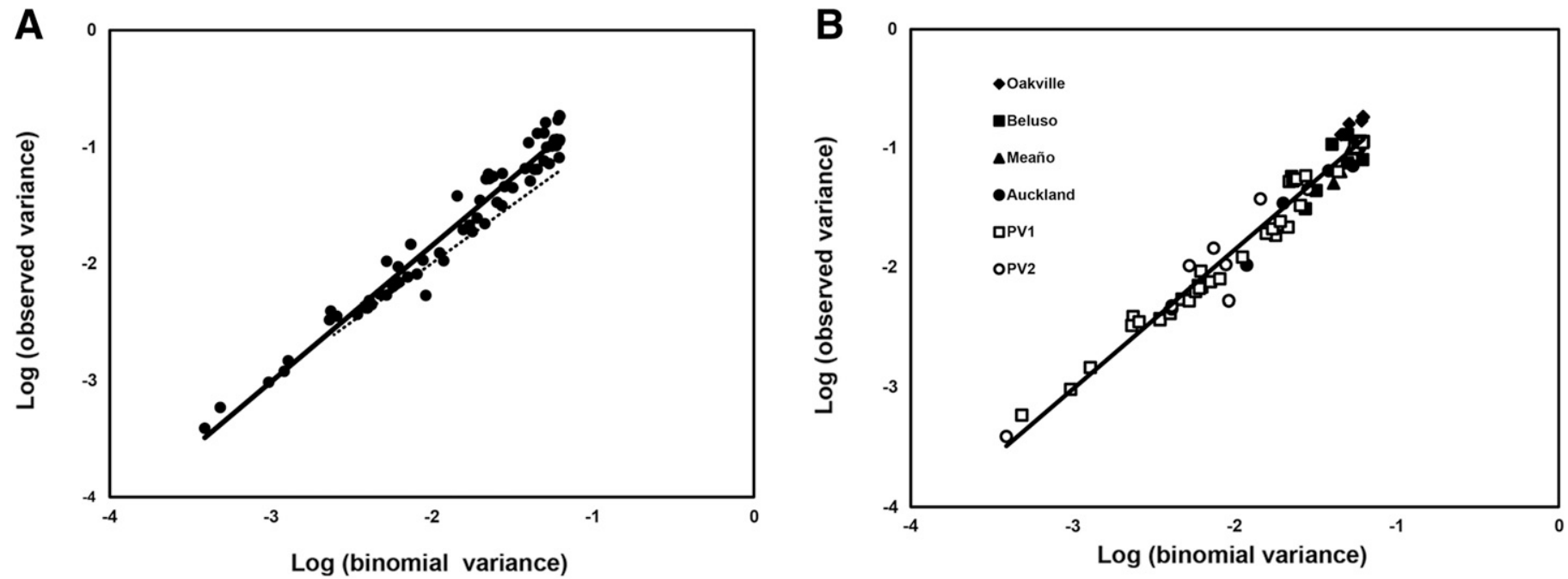

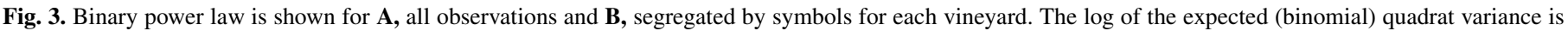

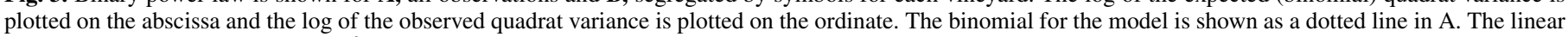
regression is $y=0.477+1.162 x\left(R^{2}=0.969, P<0.0001\right.$, which is significant $)$.
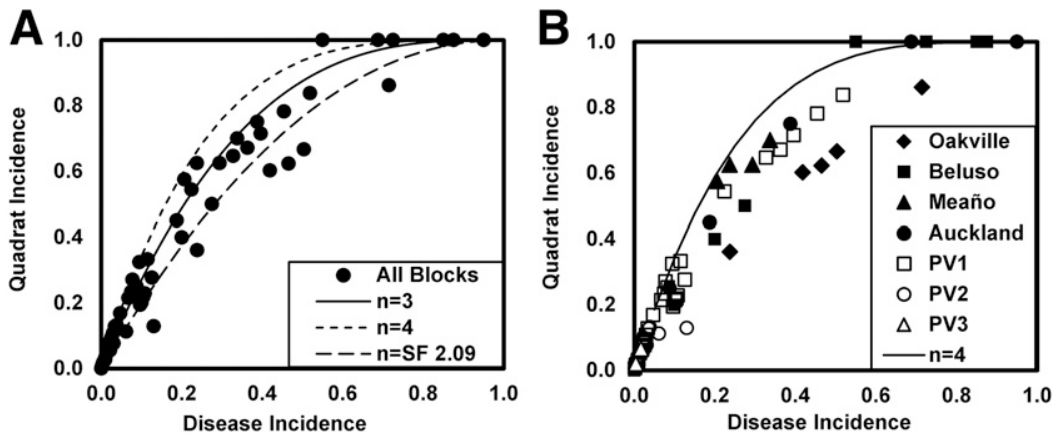

Fig. 4. Two spatial hierarchies are shown here: $I_{(l o w)}$ (disease incidence) plotted on the abscissa and $I_{(\text {high }}$ (quadrat incidence) plotted on the ordinate, with the proportion of infected clusters as $n=4$. A, All vineyard blocks are plotted as a spatial hierarchy, with the expected (binomial) distribution expressed linearly in quadrats (sample clusters) of $n=3, n=4$, and $n=2.09$, the statistical fit value produced by the complimentary $\log$-log transformation. B, Vineyards are shown segregated by symbol, with the expected (binomial) distribution expressed linearly as $n=4$. 
the previous year's newly infected vines within the vineyard block are added to the source of inoculum for the current year, allowing mealybugs which are born and feed on infected vines to transmit the virus into neighboring vines.

Old, heavily infected vineyard blocks are excellent sources of inoculum for neighboring blocks. For this reason, these blocks should be removed as soon as possible when considering planting a new, certified block nearby; illustrative examples of this situation are shown in Figures 6 and 7. Figure 6 is from previously published data (Golino et al. 2008), showing a well-developed, heavily aggregated infection from one predominant source of inoculum (block 2), while Figure 7 shows previously unpublished data illustrating invasion of a new block (B), replanted between two older blocks (A and C), resulting in inoculum sources on two sides. The neighboring blocks $\mathrm{A}$ and $\mathrm{C}$ illustrate older epidemics, with aggregated spatial patterns.

Primary inoculum levels in certified vineyards are typically low, due to the initial removal of viruses from foundation material and

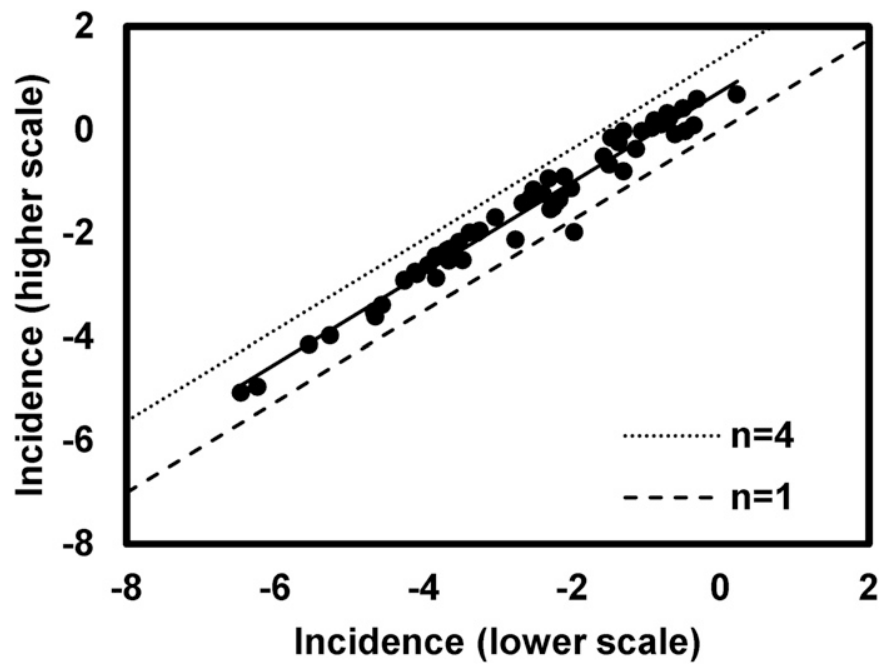

Fig. 5. Statistical fit, $n=2.09=\left(e^{0.74}\right)$, was calculated using a linear regression of the complimentary $\log -\log (\mathrm{CLL}[\bullet])$ function $y=\ln (-\ln (1-x))$ applied to both $I_{\text {(high) }}$ and $I_{\text {(low) }}$ (McRoberts et al. 2003). The linear relationship of the data are shown here with the $\operatorname{CLL}(\bullet)$ transformation of $I_{(l o w)}$ plotted on the abscissa and the CLL( $\bullet$ ) transformation of $I_{(\text {high }}$ plotted on the ordinate. The theoretical expected (binomial) distribution is plotted linearly for $n=4$ (maximum) and $n=1$ (minimum) continual diagnostic testing performed on registered nursery increase blocks, each facet being part of the CGR\&C program. When using certified rootstock and scion material, the potential for planting GLRaV-3-infected material is low. Because disease spread occurs predominantly over short spatial scales, removal of individual infected vines when disease incidence is low has a large impact on delaying the buildup of GLRaV-3 in a block. In Napa Valley, where this system has been adopted at a regional level, wine grape growers are observing a stable reduction in disease incidence in the presence of the native vector, grape mealybug (Pseudococcus maritimus).

The results presented in this article confirm that the epidemiology of GLRaV-3 is complex and cyclically polyetic. Although considerable variation is apparent in the rate of disease increase among different data sets, a common relationship relating the spatial pattern of GLRaV-3 epidemics to their mean disease incidence was revealed. In other words, GLRaV-3 epidemics develop in such a way that their spatial characteristics follow a predictable path but they do so at speeds which depend on local conditions.

Broadly, assuming a starting position of predominantly healthy planting material, initial infections tend to occur at random locations within the first few years of the vineyard's life due to small amounts of infected material entering via infected planting material, viruliferous mealybugs transmitting GLRaV-3 into uninfected vines from neighboring infected blocks, or movement of machinery, personnel, and so on. These random infections intensify into disease foci through nearest-neighbor spread of the virus by crawling mealybugs. Disease incidence grows annually in a manner that is consistent with the well-developed theoretical framework captured in the binary power law (Hughes et al. 1997; Madden and Hughes 1994; McRoberts et al. 2003). However, when viewed over a sufficiently long time scale, it is clear that secondary inoculum produced from vines infected from local sources within each block drives the dynamics of leafroll epidemics. On the other hand, the long time scale of the epidemics means that the annual removal of infected vines can lead to successful suppression of GLRaV-3, much as removal of initial inoculum can be used in the management of a monocyclic disease. However, because the longterm dynamics are driven by preexisting infections within the vineyard, GLRaV-3 can become difficult to manage if disease incidence is allowed to increase to the point where within-block infections become a significant proportion of initial inoculum each year. Recognizing that initial infections in newly planted blocks most frequently arise from neighboring, previously infected blocks, wine grape growers in California are beginning to coordinate
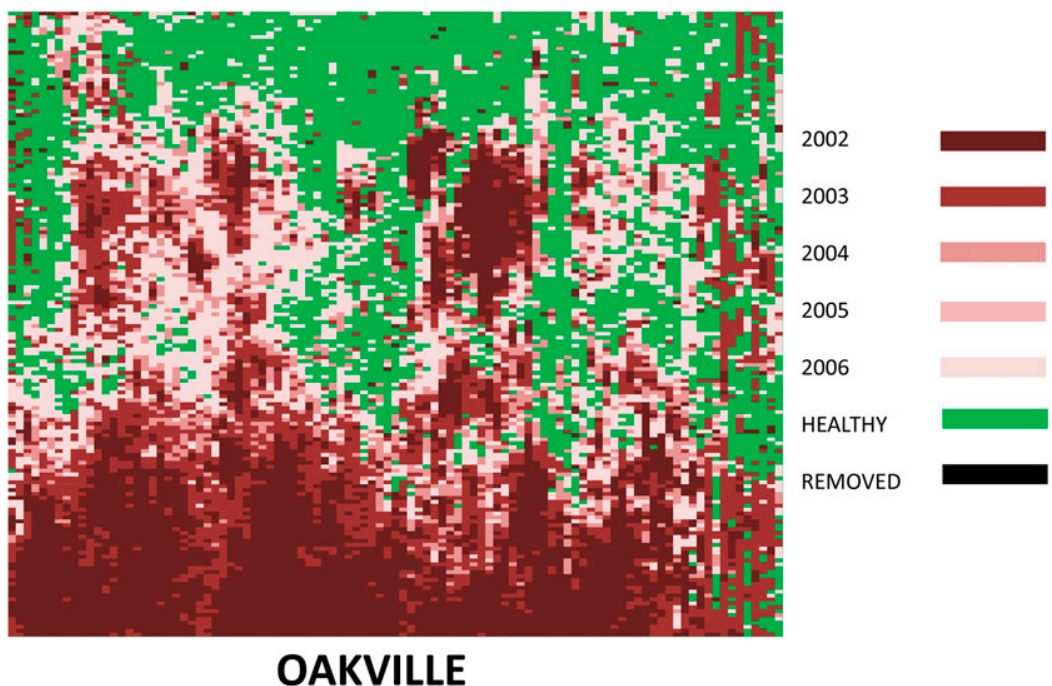

OAKVILLE

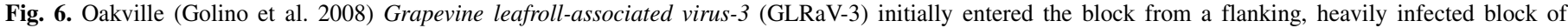
grapevines. Over time, spread within the vineyard blocks becomes the predominant source of further GLRaV-3 spread. 

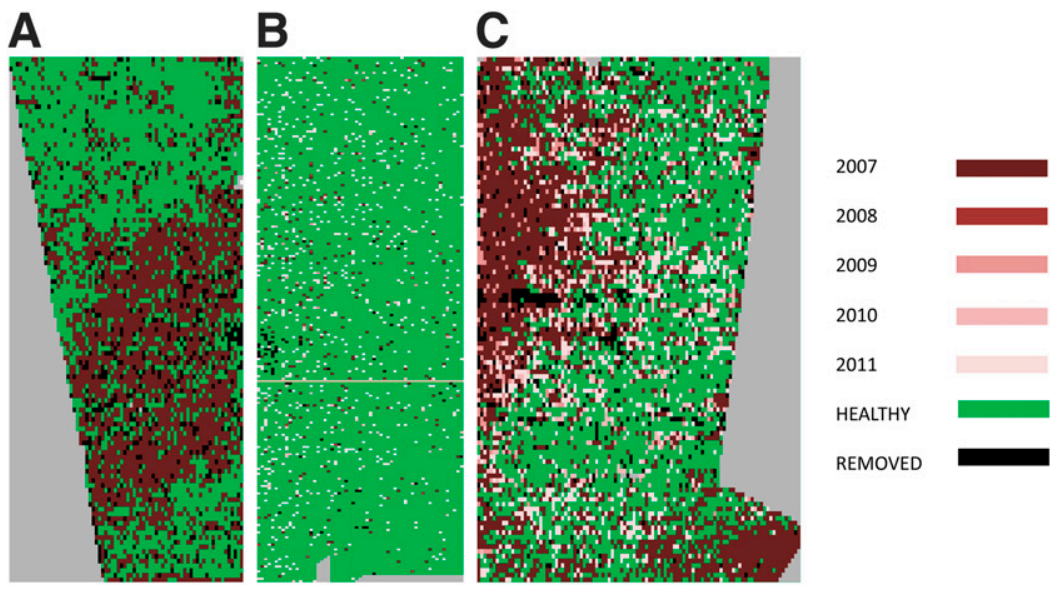

Fig. 7. Unpublished private, historic, Napa vineyard data are shown here. Block A was planted in 1989 and symptomatic vines were recorded in 2007. Block B was planted in 2002 and symptomatic vines were recorded in 2007, 2008, 2009, 2010, and 2011. Block C was planted in 1988 and symptomatic vines were recorded in 2007, 2009, 2010, and 2011.

disease management practices over neighborhoods in order to control GLRaV-3 regionally. Components of these coordinated programs include planting certified material and scouting and rogueing infected vines from certified blocks, as well as using mealybug mating disruption, timing insecticide sprays, and releasing biological controls where feasible (Almeida et al. 2013). At a more strategic level, the spatial parameters for GLRaV-3 estimated in this study are being adopted by the CGR\&C program in order to improve virus detection and testing strategies for GLRaV-3 in certified nursery increase blocks.

\section{ACKNOWLEDGMENTS}

Funding for this project was provided by the American Vineyard Foundation; the California Grape Rootstock Improvement Commission; the California Fruit Tree, Nut Tree and Grapevine Industry Advisory Board; and a historic Napa vineyard. We thank the staff, students, and faculty of Foundation Plant Services, University of California-Davis, for GLRaV-3 diagnostic services as well as providing GLRaV-3 mapping data from their previously published Oakville study. Additionally, we extend our gratitude to the census level mapping of leafroll provided by the private historic Napa vineyard which added significantly to the spatial characterization of this disease as well as the growers and vineyard managers in several California wine grape growing areas, including Lodi, Monterey, Napa, Paso Robles, and Sonoma, for many hours of useful discussion and access to vineyard blocks.

\section{LITERATURE CITED}

Alley, C., Goheen, A., Olmo, H., and Koyama, A. 1963. The effect of virus infections on vines, fruit and wines of Ruby Cabernet. Am. J. Enol. Vitic. 14:164-170.

Alley, L., and Golino, D. 2000. The origins of the grape program at Foundation Plant Materials Service. Pages 222-230 in: Proc. Am. Soc. Enol. Viticult. 50th Anniv. Meet. Seattle, WA.

Almeida, R. P., Daane, K. M., Bell, V. A., Blaisdell, G. K., Cooper, M. L., Herrbach, E., and Pietersen, G. 2013. Ecology and management of grapevine leafroll disease. Front. Microbiol. 4. doi: 10.3389/fmicb.2013.00094

Al Rwahnih, M., Dolja, V. V., Daubert, S., Koonin, E. V., and Rowhani, A. 2012. Genomic and biological analysis of Grapevine leafroll-associated virus 7 reveals a possible new genus within the family Closteroviridae. Virus Res. 163: 302-309.

Bostock, R., Thomas, C., Hoenisch, R., Golino, D., and Vidalakis, G. 2014. Plant health: How diagnostic networks and interagency partnerships protect plant systems from pests and pathogens. Calif. Agric. 68:117-124.

Burnham, K., Anderson, D., and Huyvaert, K. 2011. AIC model selection and multimodel inference in behavioral ecology: Some background, observations, and comparisons. Behav. Ecol. Sociobiol. 65:23-35.

Cabaleiro, C., Couceiro, C., Pereira, S., Cid, M., Barrasa, M., and Segura, A. 2008. Spatial analysis of epidemics of Grapevine leafroll associated virus 3. Eur. J. Plant Pathol. 121:121-130.

Cabaleiro, C., and Segura, A. 1997a. Field transmission of Grapevine leafroll associated virus-3 (GLRaV-3) by the mealybug Planococcus citri. Plant Dis. 81:283-287.
Cabaleiro, C., and Segura, A. 1997b. Some characteristics of the transmission of Grapevine leafroll associated virus-3 by Planococcus citri Risso. Eur. J. Plant Pathol. 103:373-378.

Cabaleiro, C., and Segura, A. 2006. Temporal analysis of Grapevine leafroll associated virus 3 epidemics. Eur. J. Plant Pathol. 114:441-446.

Daane, K., Almeida, R. B. V., Walker, J., Botton, M., Fallahzadeh, M., Mani, M., Miano, J., Sforza, R., Walton, V. A., and Zaviezo, T. 2012. Biology and management of mealybugs in vineyards. Pages 271-307 in: Arthropod Management in Vineyards. N. J. Bostanian, C. Vincent, and R. Isaacs, eds. Springer, Dordrecht, Heidelberg, New York, London.

Fuller, K. B., Alston, J. M., and Golino, D. A. 2015. The economic benefits from virus-screening: A case study of grapevine leafroll in the north coast of California. Am. J. Enol. Vitic. 66:112-119.

Gambino, G., Bondaz, J., and Gribaudo, I. 2006. Detection and elimination of viruses in callus, somatic embryos and regenerated plantlets of grapevine. Eur. J. Plant Pathol. 114:397-404.

Goheen, A., and Cook, J. A. 1959. Leafroll (red-leaf or rougeau) and its effects on vine growth, fruit quality, and yields. Am. J. Enol. Vitic. 10:173-181.

Goheen, A., Hewitt, W., and Alley, C. 1959. Studies of grape leafroll in California. Am. J. Enol. Vitic. 10:78-84.

Golino, D., Sim, S., Gill, R., and Rowhani, A. 2002. California mealybugs can spread grapevine leafroll disease. Calif. Agric. 56:196-201.

Golino, D., Weber, E., Sim, S., and Rowhani, A. 2008. Leafroll disease is spreading rapidly in a Napa Valley vineyard. Calif. Agric. 62:156-160.

Habili, N., and Nutter, F. W. 1997. Temporal and spatial analysis of Grapevine leafroll-associated virus 3 in Pinot Noir grapevines in Australia. Plant Dis. $81: 625-628$.

Hale, C., and Woodham, R. 1979. Effect of grapevine leafroll disease on the acid and potassium composition of Sultana grapes. Am. J. Enol. Vitic. 30:91-92.

Hewitt, W. B., Goheen, A., Raski, D., and Gooding, G. 1962. Studies on virus diseases of the grapevine in California. Vitis 3:57-83.

Hughes, G., and Gottwald, T. R. 1999. Survey methods for assessment of Citrus tristeza virus incidence when Toxoptera citricida is the predominant vector. Phytopathology 89:487-494.

Hughes, G., and Madden, L. 1992. Aggregation and incidence of disease. Plant Pathol. 41:657-660.

Hughes, G., and Madden, L. 1993. Using the beta-binomial distribution to describe aggregated patterns of disease incidence. Phytopathology 83:759-763.

Hughes, G., Madden, L., and Munkvold, G. 1996. Cluster sampling for disease incidence data. Phytopathology 86:132-137.

Hughes, G., and McRoberts, N. 2014. The structure of diagnostic information. Australas. Plant Pathol. 43:267-286.

Hughes, G., McRoberts, N., Madden, L. V., and Gottwald, T. R. 1997. Relationships between disease incidence at two levels in a spatial hierarchy. Phytopathology 87:542-550.

Jordan, D. 1993. Leafroll spread in New Zealand vineyards. Aust. N. Z. Wine Ind. J. 8:322-324.

Klaassen, V. A., Sim, S. T., Dangl, G., Osman, F., Al Rwahnih, M. R. A., and Golino, D. 2011. Vitis californica and Vitis californica $\times$ Vitis vinifera hybrids are hosts for Grapevine leafroll-associated virus-2 and -3 and Grapevine virus $A$ and B. Plant Dis. 95:657-665.

Lee, J., and Martin, R. R. 2009. Influence of grapevine leafroll associated viruses (GLRaV-2 and-3) on the fruit composition of Oregon Vitis vinifera L. cv. Pinot noir. Phenolics Food Chem. 112:889-896. 
Lider, L., Goheen, A., and Ferrari, N. 1975. A comparison between healthy and leafroll-affected grapevine planting stocks. Am. J. Enol. Vitic. 26: 144-147.

Madden, L. V., and Hughes, G. 1994. BBD_Computer software for fitting the beta-binomial distribution to disease incidence data. Plant Dis. 78:536-540.

Madden, L. V., and Hughes, G. 1995. Plant disease incidence: Distributions, heterogeneity, and temporal analysis. Annu. Rev. Phytopathol. 33:529564.

Madden, L. V., Hughes, G., and van den Bosch, F., eds. 2007. The Study of Plant Disease Epidemics. American Phytopathological Society Press, St. Paul, MN.

Maree, H. J., Almeida, R. P. P., Bester, R., Chooi, K. M., Cohen, D., Dolja, V. V., Fuchs, M. F., Golino, D. A., Jooste, A. E. C., Martelli, G. P., Naidu, R. A., Rowhani, A., Saldarelli, P., and Burger, J. T. 2013. Grapevine leafrollassociated virus 3. Front. Microbiol. 4. doi: 10.3389/fmicb.2013.00082

Maree, H. J., Pirie, M. D., Oosthuizen, K., Bester, R., Rees, D. J. G., and Burger, J. T. 2015. Phylogenomic analysis reveals deep divergence and recombination in an economically important grapevine virus. PLoS One 10: e0126819.

Martelli, G., Abou Ghanem-Sabanadzovic, N., Agranovsky, A., Al Rwahnih, M., Dolja, V., Dovas, C., Fuchs, M., Gugerli, P., Hu, J., and Jelkmann, W. 2012. Taxonomic revision of the family Closteroviridae with special reference to the grapevine leafroll-associated members of the genus Ampelovirus and the putative species unassigned to the family. J. Plant Pathol. 94: 7-19.

McRoberts, N., Hughes, G., and Madden, L. 2003. The theoretical basis and practical application of relationships between different disease intensity measurements in plants. Ann. Appl. Biol. 142:191-211.

Naidu, R. A., Maree, H. J., and Burger, J. T. 2015. Grapevine leafroll disease and associated viruses: A unique pathosystem. Annu. Rev. Phytopathol. 53: 613-634.
Olmo, H. P. 1951. A proposed program for the introduction improvement and certification of healthy grape varieties. Wines Vines 32:7-9.

Osman, F., Leutenegger, C., Golino, D., and Rowhani, A. 2007. Real-time RT-PCR (TaqMan) assays for the detection of Grapevine leafroll associated viruses 1-5 and 9. J. Virol. Methods 141:22-29.

Osman, F., and Rowhani, A. 2008. Real-time RT-PCR (TaqMan) assays for the detection of viruses associated with rugose wood complex of grapevine. J. Virol. Methods 154:69-75.

Panattoni, A., and Triolo, E. 2010. Susceptibility of grapevine viruses to thermotherapy on in vitro collection of Kober 5BB. Sci. Hortic. (Amsterdam Neth.) 125:63-67.

Ricketts, K. D., Gomez, M. I., Atallah, S. S., Fuchs, M. F., Martinson, T. E., Battany, M. C., Bettiga, L. J., Cooper, M. L., Verdegaal, P. S., and Smith, R. J. 2015. Reducing the economic impact of grapevine leafroll disease in California: Identifying optimal disease management strategies. Am. J. Enol. Viticult. 66:138-147.

Seah, Y. M., Sharma, A. M., Zhang, S., Almeida, R. P. P., and Duffy, S. 2012. A divergent variant of Grapevine leafroll-associated virus 3 is present in California. Virol. J. 9:235.

Sharma, A. M., Wang, J., Duffy, S., Zhang, S., Wong, M. K., Rashed, A., Cooper, M. L., Daane, K. M., and Almeida, R. P. 2011. Occurrence of grapevine leafroll-associated virus complex in Napa Valley. PLoS One 6:e26227.

Tsai, C.-W., Chau, J., Fernandez, L., Bosco, D., Daane, K., and Almeida, R. 2008. Transmission of Grapevine leafroll-associated virus 3 by the vine mealybug (Planococcus ficus). Phytopathology 98:1093-1098.

Tsai, C.-W., Rowhani, A., Golino, D. A., Daane, K. M., and Almeida, R. P. 2010. Mealybug transmission of grapevine leafroll viruses: An analysis of virus-vector specificity. Phytopathology 100:830-834.

Turechek, W. W., and McRoberts, N. 2013. Considerations of scale in the analysis of spatial pattern of plant disease epidemics. Annu. Rev. Phytopathol. 51:453-472. 